\title{
Functional neuroimaging of amphetamine-induced striatal neurotoxicity in the pleiotrophin knockout mouse model
}

\section{Authors:}

María Luisa Soto-Montenegro1,2,*, Marta Vicente-Rodríguez ${ }^{4}$, Carmen

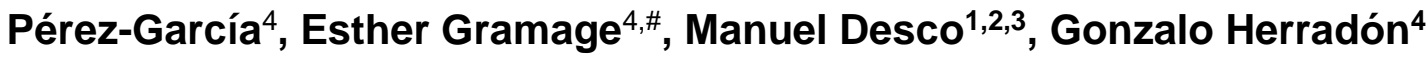

${ }^{1}$ Unidad de Medicina y Cirugía Experimental, Instituto de Investigación Sanitaria Gregorio Marañón, Madrid, Spain

${ }^{2}$ CIBER de Salud Mental (CIBERSAM), Madrid, Spain

${ }^{3}$ Departamento de Bioingeniería e Ingeniería Aeroespacial, Universidad Carlos III de Madrid, Spain

${ }^{4}$ Laboratorio de Farmacología, Departamento de Ciencias Farmacéuticas y de la Salud, Facultad de Farmacia, Universidad CEU San Pablo, Madrid, Spain

\#Current address: Department of Ophthalmology and Visual Sciences, W. K. Kellogg Eye Center, University of Michigan Medical School, Ann Arbor, Michigan, USA

Running title: Functional imaging of striatal damage

Conflicts of interest: The authors declare no conflicts of interest.

\section{*Corresponding author:}

ML Soto-Montenegro, PhD

Unidad de Medicina y Cirugía Experimental

Hospital General Universitario Gregorio Marañon

Dr. Esquerdo, 46

E-28007 Madrid, Spain

Fax \#: +34914265108

Phone \#: +3491586 6678

e-mail: marisa@mce.hggm.es 


\section{ABSTRACT}

Amphetamine-induced neurotoxic effects have traditionally been studied using immunohistochemistry and other post-mortem techniques, which have proven invaluable for the definition of amphetamine-induced dopaminergic damage in the nigrostriatal pathway. However, these approaches are limited in that they require large numbers of animals and do not provide the temporal data that can be collected in longitudinal studies using functional neuroimaging techniques. Unfortunately, functional imaging studies in rodent models of druginduced neurotoxicity are lacking. The aim of this study was to evaluate in vivo the changes in brain glucose metabolism caused by amphetamine in the pleiotrophin knockout mouse (PTN-/-), a genetic model with increased vulnerability to amphetamine-induced neurotoxic effects. We showed that administration of amphetamine causes a significantly greater loss of striatal tyrosine hydroxylase content in PTN-/- mice than in wild-type (WT) mice. In addition, $\left[{ }^{18} \mathrm{~F}\right]-\mathrm{FDG}-\mathrm{PET}$ shows that amphetamine produces a significant decrease in glucose metabolism in the striatum and prefrontal cortex in the PTN-/- mice, compared to WT mice. These findings suggest that $\left[{ }^{18} \mathrm{~F}\right]-\mathrm{FDG}$ uptake measured by PET is useful for detecting amphetamine-induced changes in glucose metabolism in vivo in specific brain areas, including the striatum, a key feature of amphetamine-induced neurotoxicity.

KEYWORDS: [18F]-FDG-PET, amphetamine, pleiotrophin, neurotoxicity, Parkinson's disease, midkine. 


\section{INTRODUCTION}

Amphetamine and its derivatives are widespread drugs of abuse that lead to addictive behavior and severe neurological damage [7, 39]. As a drug of abuse, amphetamine activates the reward pathway of the brain. Most of the mesolimbic and mesocortical projections involved in reward are located in the ventral tegmental area. Its dopaminergic neurons send their axons to nucleus accumbens, the striatum and prefrontal cortex. On the other hand, hallmarks of neurotoxicity induced by these drugs include proliferation of astrocytes in striatum [19], apoptosis of striatal neurons, and destruction of striatal dopaminergic terminals [2, 19]. In addition, amphetamine derivatives have been shown to induce dopaminergic cell loss in the substantia nigra, which is also a feature of Parkinson's disease (PD) [11]. The prevalence of PD was recently shown to be increased in users of amphetamine-type drugs [5], suggesting that these drugs increase the risk of developing PD. However, the authors also showed that a high proportion of the 40,000 methamphetamine users enrolled did not develop PD during the 15 years of the study, thus suggesting the possible existence of genetic factors underlying individual vulnerability to PD after consumption of this type of psychostimulants. These genetic factors include the neurotrophic factor pleiotrophin (PTN), which has recently received significant attention [12]. PTN has been shown to limit the neurotoxic effects of amphetamine $[9,10]$. Accordingly, genetic inactivation of PTN confers increased vulnerability to amphetamine-induced neurotoxic effects in the nigrostriatal pathway. Administration of amphetamine in mice that are genetically deficient in PTN (PTN-/-) results in increased astrocytosis in the striatum and substantia nigra, increased loss of striatal dopaminergic terminals, 
and, surprisingly, loss of tyrosine hydroxylase (TH)-positive neurons in the substantia nigra, an effect that is not observed in WT mice [10]. These different responses to amphetamine in PTN-/- and WT mice suggest this model could be useful for identifying the remaining unknown mechanisms through which amphetamine exerts its neurotoxic effects and for identifying druggable targets within those mechanisms [12].

Traditional studies characterizing amphetamine-induced neurotoxic effects are subject to significant limitations that prevent more efficient approaches. Immunohistochemistry and other post-mortem techniques have proven invaluable in our investigations of amphetamine-induced dopaminergic damage in the nigrostriatal pathway. However, they require large numbers of animals and do not provide the temporal data that can be collected in longitudinal studies. Functional neuroimaging techniques, such as positron emission tomography (PET), single-photon emission computed tomography, functional magnetic resonance imaging (fMRI), and electro-encephalography, have been extensively applied to study the emotional and cognitive-behavioral components of addiction [27]. These techniques are ideally suited for studying the potential neurotoxic effects of drugs in the living brain and make it possible to characterize the functional effects of parkinsonian toxins in the rodent brain [16]. MRI has been used in humans to investigate in vivo tissue abnormalities caused by the neurotoxic effects of drugs [36]; however, functional imaging studies in animal models are lacking. In this study, we used the PTN-/- mouse, a genetic model with increased vulnerability to amphetamine-induced neurotoxic effects, to validate $\left[{ }^{18} \mathrm{~F}\right]-$ fluoro-2-deoxy-d-glucose $\left(\left[{ }^{18} \mathrm{~F}\right]-\mathrm{FDG}\right)$ PET as a useful neuroimaging marker of neurotoxicity in mice. 


\section{MATERIAL AND METHODS}

PTN-/-mice

PTN-/- mice were generated as previously described [1]. We used male PTN-/and $\mathrm{PTN}+++(\mathrm{WT})$ animals aged 9-10 weeks (20-25 g). All experimental animal procedures complied with European Union Council Directive 2010/63/EU and were approved by the Institutional Animal Care and Use Committee.

\section{$\underline{\text { Drug protocol }}$}

Animals received 4 consecutive intraperitoneal injections of amphetamine $(10 \mathrm{mg} / \mathrm{kg})$ or saline $(10 \mathrm{ml} / \mathrm{kg})$ with an interval of 2 hours between administrations. This "binge" regimen is known to cause significant damage to striatal dopaminergic terminals [20]. For both immunohistochemistry $(\mathrm{IH})$ and PET imaging studies, mice were divided into 4 groups according to the drug protocol applied: A) PTN-/ + saline (PTN-/- control); B) PTN-/- + amphetamine; C) WT + saline (WT control); and D) WT + amphetamine.

Our study sample comprised 22 PTN-/- and 22 WT mice. The animals used for the IH studies were different from those used in the PET studies in order to test for striatal TH loss $(\mathrm{IH})$ and FDG uptake (PET) at the same time point after the last amphetamine or saline administration. The sample size for both IH and PET studies was 5-6 animals per group.

\section{Immunohistochemistry studies}

One of the consequences of amphetamine administration is the loss of dopaminergic terminals in the striatum [3]. We previously demonstrated significantly greater striatal dopaminergic denervation in PTN-/- than in WT mice [8]. In confirmation of those studies, and as part of the validation process of $\left[{ }^{18} \mathrm{~F}\right]-\mathrm{FDG}-\mathrm{PET}$ to assess the neurotoxic effects of amphetamine, expression of 
TH in the striatum of PTN-/- and WT mice treated with either amphetamine or saline was analyzed using immunohistochemistry.

Animals were euthanized 4 days after the last administration of amphetamine or saline because at this time point we have previously found significant between-genotype differences in amphetamine-induced striatal $\mathrm{TH}$ loss [9]. The brains were removed immediately and conserved in $p$ formaldehyde for 7 days before being transferred to a solution of $0.1 \mathrm{M}$ phosphate buffer containing $0.02 \%$ sodium azide for storage at $4^{\circ} \mathrm{C}$. The brains were cut into $30 \mu \mathrm{m}$ sections using a vibratome (Leica, Wetzlar, Germany), and striatal free-floating sections were processed following a protocol previously described [9]. Striatal TH-positive fiber staining was assessed using optical density (OD) measurements after digitalized images of $\mathrm{TH}$-immunostained striatal sections were collected. ODs were measured using Image-Pro Plus software (Version 3.0.1; Media Cybernetics, Silver Spring, Maryland, USA). For each animal, the nonspecific background correction in each section was made by subtracting the $O D$ value of the corpus callosum from the striatal $O D$ value obtained from the same section.

\section{$\underline{\text { PET-CT imaging studies }}$}

All animals were scanned 4 days after the end of the drug administration protocol using a small-animal PET/CT scanner (ARGUS, SEDECAL, Madrid, Spain) under isoflurane anesthesia (3\% induction and $1.5 \%$ maintenance in $100 \% \mathrm{O}_{2}$ ). This anesthesia was used because of its lower effect on brain glucose metabolism compared to other anesthetics [32], although the ideal situation would have been to perform the imaging study at consciousness [25]. $\left[{ }^{18} \mathrm{~F}\right]-\mathrm{FDG}(\sim 0.5 \mathrm{mCi})$ was injected into the tail vein, and, after an uptake period 
of 45 minutes, animals were imaged for 60 minutes. Images were reconstructed using a 2D-OSEM algorithm. The spatial resolution for the scanner was 1.45 $\mathrm{mm}$ full width at half maximum (FWHM). The energy window was $400-700 \mathrm{keV}$. Decay and deadtime corrections were applied.

Computed tomography (CT) studies were acquired using the abovementioned PET/CT scanner. The images were reconstructed using a Feldkamp algorithm. These anatomical images did not require registration with their corresponding PET scans thanks to the intrinsic alignment of the PET/CT device.

\section{MRI study}

An MRI study of one WT mouse was acquired with a 7-Tesla Biospec 70/20 scanner (Bruker, Ettlingen, Germany). The animal was anesthetized with sevoflurane $\left(4.5 \%\right.$ induction and $2.5 \%$ maintenance in $\left.100 \% \mathrm{O}_{2}\right)$. A T2weighted spin echo sequence was acquired with $\mathrm{TE}=33 \mathrm{~ms}, \mathrm{TR}=4281 \mathrm{~ms}$, and a slice thickness of $0.4 \mathrm{~mm}$ (33 slices). Matrix size was $256 \times 256$ pixels at an FOV of $1.6 \times 1.6 \mathrm{~cm}^{2}$. The inhomogeneity artifact caused by the use of the surface antenna was corrected.

This single study was used as an anatomical template for the PET images.

\section{Analysis of PET data}

PET data were analyzed using regions of interest (ROI). All CT studies were automatically co-registered with a reference CT scan that was chosen randomly from among the animals [28]. Since the PET/CT small animal scanner provides aligned CT and PET images for each animal, the spatial transformation obtained for each CT image was subsequently applied to the 
corresponding PET image. The ROIs drawn on coronal sections in the MRI image were striatum, prefrontal cortex, thalamus, cerebellum (CB), background, and whole brain (WB). Two regions of reference (ROlref) were used to normalize $\left[{ }^{18} \mathrm{~F}\right]-\mathrm{FDG}$ uptake, the whole brain (ratio to $\mathrm{WB}$ ) and the cerebellum (ratio to CB). WB is the preferred reference region in most studies with small animals [32, 34, 35] (provided there are no between-group differences in this region [6], as it is our case). The PET data assessment included the analysis of mean Standardized Uptake Value (SUV), which takes into account the tissue radioactivity concentration, the injected activity and the body weight.

\section{Statistical analysis}

Data from $\mathrm{IH}$ and PET studies are presented as mean \pm standard deviation (SD). The hypothesis of normality of the distributions and homogeneity of variance were assessed using the Kolmogorov-Smirnov and Levene tests, respectively. Intra-subject variability was assessed through the coefficient of variation (COV), which shows a range of $15-30 \%$ in other studies on neurodegenerative disorders characterized by striatal dopaminergic damage [14]. Data were analyzed using one-way analysis of variance (ANOVA) followed by post hoc tests (Tukey). All statistical analyses were performed using the SPSS 15.0 software package.

The threshold for statistical significance was set at $p<0.05$.

\section{RESULTS}

\section{Immunohistochemistry}

ANOVA of TH expression in the striatum revealed a significant group effect $(F=30.68, p<0.0001$, d.f. $=3)$. The striatal $\mathrm{TH}$ levels in amphetamine- 
treated WT animals $(0.49 \pm 0.07$ arbitrary units [au]) were significantly lower than those of WT controls $(0.90 \pm 0.06 \mathrm{au})(p<0.01)$ (Fig. 1A). The striatal TH levels in amphetamine-treated PTN-/- animals $(0.18 \pm 0.04 \mathrm{au})$ were also significantly lower than those of PTN-/- controls $(0.84 \pm 0.09 \mathrm{au})(p<0.001)$. Interestingly, we also found that striatal TH levels in amphetamine-treated PTN-/- mice were significantly lower than those in amphetamine-treated WT mice, whereas TH levels in control animals were similar in both genotypes (Fig. 1A).

\section{PET}

Measurements based on absolute value of SUV for each ROI resulted in no significant statistical differences between groups (Fig. 1C), probably due to the high COV (range: 5-30\%). When PET data were normalized, COV decreased to $2-9 \%$, thus allowing us to detect statistically significant differences. We performed the analysis of $\left[{ }^{18} \mathrm{~F}\right]-\mathrm{FDG}$ uptake data using two ROlref for normalization, the WB and $\mathrm{CB}$. One pre-requisite to use $\mathrm{WB}$ as the reference region is to make sure there are no between-group differences in global uptake [6], as it is our case. The tendency with both normalizations was similar, although statistical significance was achieved when normalizing to WB. We decided to use WB as our ROlref, as it is the case in most studies with small animals $[32,34,35]$.

ANOVA of $\left[{ }^{18} \mathrm{~F}\right]-\mathrm{FDG}$ uptake in the prefrontal cortex and striatum revealed a significant group effect $(F=4.924, p=0.011$, d.f. $=3$ and $F=6.747$, $p=0.003$, d.f. $=3$ respectively). No statistically significant between-group differences were found in the thalamus and cerebellum (Fig. 1B,C).

Striatum: Uptake in amphetamine-treated PTN-/- animals $(1.12 \pm 0.06)$ was significantly lower than in amphetamine-treated WT mice $(1.27 \pm 0.08)$ 
$(p=0.009) \quad$ (Fig. 1B,C). We also found that striatal $\left[{ }^{18} \mathrm{~F}\right]-\mathrm{FDG}$ uptake in amphetamine-treated PTN-/- mice was significantly lower compared with salinetreated WT mice $(p=0.004)$, whereas $\left[{ }^{18} \mathrm{~F}\right]-\mathrm{FDG}$ uptake in control animals was relatively similar in both genotypes (Fig. 1B,C).

Prefrontal cortex: $\left[{ }^{18} \mathrm{~F}\right]-\mathrm{FDG}$ uptake in amphetamine-treated PTN-/- mice $(1.02 \pm 0.06)$ was significantly lower than in amphetamine-treated WT mice $(1.16 \pm 0.05)(p=0.012)$, whereas $\left[{ }^{18} \mathrm{~F}\right]-\mathrm{FDG}$ uptake in control animals was relatively similar in both genotypes (Fig. 1B,C).

\section{DISCUSSION}

To our knowledge, this is the first report to demonstrate that striatal damage caused by amphetamine treatment correlates with significantly decreased striatal $\left[{ }^{18} \mathrm{~F}\right]-\mathrm{FDG}$ uptake in PTN-/- mice and can therefore be assessed using $\left[{ }^{18} \mathrm{~F}\right]-\mathrm{FDG}-\mathrm{PET}$. Also, a significant decrease in glucose metabolism was found in the prefrontal cortex in amphetamine-treated PTN-/mice compared to WT mice.

Despite existing evidence based on the results of imaging techniques in humans $[31,36]$, few studies have used $\left[{ }^{18}\right.$ F]-FDG-PET to explore the effects of drugs in animal models [13, 35]. This gap in knowledge is significant, considering that the most remarkable advances in the characterization of the mechanisms underlying psychostimulants-induced neurotoxicity have been achieved in rodent models. In addition, these models have enabled identification of critical genetic factors modulating the toxic effects of psychostimulants and potential pharmacological targets to prevent the brain damage they induce [12]. 
The recent development of PET scanners for laboratory animals enables detailed in vivo study of the neurotoxic effects of drugs in rodent models by providing insights into the biochemical and molecular processes involved and noninvasive follow-up with neuromodulatory approaches [35]. Because of the close relationship between energy expenditure (glucose metabolism) and functional activity, PET may provide an alternative way to explore the underlying mechanisms of amphetamine induced neurotoxicity using the minimum number of animals [15]. In the present study, $\left[{ }^{18} \mathrm{~F}\right]-\mathrm{FDG}$ was used as a marker of cerebral glucose consumption resulting from neuronal entrapment and accumulation of $\left[{ }^{18} \mathrm{~F}\right]-\mathrm{FDG}-6-\mathrm{PO} 4$, which indicates neuronal activity [32]. $\left[{ }^{18} \mathrm{~F}\right]-$ FDG can be considered a broad-spectrum tracer, since its uptake in the brain is by all existing cell types, independently of the neurotransmission system involved. Thus, $\left[{ }^{18} \mathrm{~F}\right]-\mathrm{FDG}$ is a good candidate tracer for screening when the neurotransmission system affected is not known. Another advantage of $\left[{ }^{18} \mathrm{~F}\right]-$ FDG is that it has been efficiently applied in humans to demonstrate changes in relative regional cerebral glucose metabolism in users of this type of psychostimulant $[17,18,23,38]$. Finally, the much wider availability of FDG (as compared to more specific dopaminergic tracers) makes validation of this tracer more likely to become useful in clinical medicine and animal research. Our $\left[{ }^{18} \mathrm{~F}\right]-$ FDG PET data showed a slight, statistically non-significant, decrease in striatal and prefrontal cortex glucose metabolism in the WT mice, whereas glucose uptake was significantly reduced in the striatum and prefrontal cortex of PTN-/mice.

Cortico-striato-thalamic Pathway 
The $\left[{ }^{18} \mathrm{~F}\right]-\mathrm{FDG}$ method enables simultaneous visualization of metabolic changes throughout the entire nervous system, making it possible the identification of complex neuronal circuits which mediate the response to a pharmacological manipulation, similarly to $\left[{ }^{14} \mathrm{C}\right]$-deoxyglucose [33]. In this respect, we found changes in $\left[{ }^{18} \mathrm{~F}\right]-\mathrm{FDG}$ uptake in a different brain area in addition to the striatum, which highlights that not only dopamine cells are affected, as other authors have already pointed out [38]. The prefrontal cortex is part of the mesolimbic system and crucial for drug reward. Its dopaminergic innervation is limited compared to striatum. The thalamus, with poor dopaminergic innervation, is an important component of the cortico-striatothalamo-cortical loops. It constitutes a central link between the basal ganglia (striatum) and cerebral cortices and mediates information flow between cortical networks [24]. Imaging studies in methamphetamine abstinents have provided evidence of functional abnormalities in the thalamus and frontal cortex [37, 38, 40]. Even a dose-dependent frontal hypometabolism has been described in methamphetamine abusers [18]. Kim and colleagues found significantly lower metabolism in the prefrontal cortex in methamphetamine abusers, which was associated to frontal executive dysfunction $[17,18]$.

\section{Striatum}

$\left[{ }^{18} \mathrm{~F}\right]-\mathrm{FDG}$ results in the striatum are consistent with those of previous studies by our group [10] where we detected moderate depletion of TH content $(\sim 40 \%)$ in the striatum of amphetamine-treated WT mice compared with salinetreated WT mice, and a significant loss in TH content $(\sim 75 \%)$ in the striatum of amphetamine-treated PTN-/- mice compared with saline-treated PTN-/- mice. Overall, the amphetamine-induced changes in striatal glucose metabolism 
presented here are consistent with the trend in the most well-established amphetamine-induced neurotoxic effect in animal models, namely, striatal $\mathrm{TH}$ loss $[10,23]$.

Although striatal glucose metabolism might be expected to dramatically decrease after administration of amphetamine owing to reduced dopaminergic activity in the nigrostriatal pathway [21], it is important to note that other factors, such as neuroinflammation, could compensate for this reduction, since $\left[{ }^{18} \mathrm{~F}\right]-$ FDG uptake increases in neuroinflammation processes [29]. In this respect, a correlation has been found between $\left[{ }^{18} \mathrm{~F}\right]-\mathrm{FDG}$ and $\left[{ }^{11} \mathrm{C}\right]-\mathrm{PK} 11195$, a marker of activated microglia, in the seizure focus of patients with epilepsy [4]. Microglia acts as the resident macrophages in brain tissue and represents the initial responder to tissue damage or foreign pathogens. Activated microglia releases a variety of cytotoxic compounds intended to attack these pathogens, but which may also cause neuronal damage. Although methamphetamine abuse leads to the most profound neurotoxicity in the dopamine-rich basal ganglia [37], it also increases proliferation of cortical microglia and astrocytes [22], which in turn may increase the global cerebral metabolic rate [30]. Therefore, Volkow et al. [38] suggested that higher parietal activity in methamphetamine abusers could reflect reactive gliosis after excitotoxic damage to parietal neurons.

In addition, amphetamine treatment induces the appearance of reactive glial cells and astrocytes in the striatum of PTN-/- mice, as we showed in a previous study by our group [9]. Interestingly, the number of glial fibrillary acidic protein (GFAP)-positive astrocytes in the striata was higher after amphetamine treatment in PTN-/- compared with WT mice, and also the astrocytes in the PTN-/- mice developed large densely stained cell bodies and long extensive 
processes compared to the WT mice. These findings could mask a more significant decrease in glucose uptake from dopaminergic neurons than that reflected in the PET assays. Nevertheless, it is noteworthy that the amphetamine-induced decrease in striatal glucose metabolism in our murine model is quantitatively comparable to that observed in the striatum of human addicts to this type of psychostimulant [38]. Volkow and colleagues found significantly lower metabolism in the striatum ( 10\%) of abstinent methamphetamine users [38], similar to that obtained by us in the mice

striatum. Reduced striatum and amygdala $\left[{ }^{18} \mathrm{~F}\right]-\mathrm{FDG}$ uptake was observed in ecstasy users when compared to controls [26]. In this regard, our data suggest that $\left[{ }^{18} \mathrm{~F}\right]-\mathrm{FDG}$ PET may be useful for detecting amphetamine-induced striatal damage in longitudinal studies in mice.

\section{CONCLUSION}

Our findings support the use of PET for testing of $\left[{ }^{18} \mathrm{~F}\right]-\mathrm{FDG}$ uptake to evaluate amphetamine-induced damage in vivo in specific brain areas, including the striatum, in rodent models. PET could help to overcome the limitations of more traditional approaches by making it possible to perform longitudinal studies in reduced numbers of animals.

Acknowledgments: This work was supported by FIS PI14/00860, PI11/00616, PI10/02986 and ARTEMIS S2009/DPI-1802.. 


\section{REFERENCES}

[1] L.E. Amet, S.E. Lauri, A. Hienola, S.D. Croll, Y. Lu, J.M. Levorse, B. Prabhakaran, T. Taira, H. Rauvala, T.F. Vogt, Enhanced hippocampal long-term potentiation in mice lacking heparin-binding growth-associated molecule, Mol Cell Neurosci 17 (2001) 1014-1024.

[2] S. Ares-Santos, N. Granado, I. Oliva, E. O'Shea, E.D. Martin, M.I. Colado, R. Moratalla, Dopamine $\mathrm{D}(1)$ receptor deletion strongly reduces neurotoxic effects of methamphetamine, Neurobiol Dis 45 (2012) 810-820.

[3] J.F. Bowyer, L.T. Frame, P. Clausing, K. Nagamoto-Combs, C.A. Osterhout, C.R. Sterling, A.W. Tank, Long-term effects of amphetamine neurotoxicity on tyrosine hydroxylase mRNA and protein in aged rats, J Pharmacol Exp Ther 286 (1998) 1074-1085.

[4] T. Butler, M. Ichise, A.F. Teich, E. Gerard, J. Osborne, J. French, O. Devinsky, R. Kuzniecky, F. Gilliam, F. Pervez, F. Provenzano, S. Goldsmith, S. Vallabhajosula, E. Stern, D. Silbersweig, Imaging inflammation in a patient with epilepsy due to focal cortical dysplasia, J Neuroimaging 23 (2013) 129-131.

[5] R.C. Callaghan, J.K. Cunningham, J. Sykes, S.J. Kish, Increased risk of Parkinson's disease in individuals hospitalized with conditions related to the use of methamphetamine or other amphetamine-type drugs, Drug Alcohol Depend 120 (2012) 35-40.

[6] K.J. Friston, J. Ashburner, C.D. Frith, J.B. Poline, J.D. Heather, R.S.J. Frackowiak, Spatial Registration and Normalization of Images, Human Brain Mapping (1995) 165-189.

[7] E. Gramage, G. Herradon, Connecting Parkinson's disease and drug addiction: common players reveal unexpected disease connections and novel therapeutic approaches, Curr Pharm Des 17 (2011) 449-461.

[8] E. Gramage, G. Herradon, Genetic deletion of pleiotrophin leads to disruption of spinal nociceptive transmission: evidence for pleiotrophin modulation of morphine-induced analgesia, Eur J Pharmacol 647 (2010) 97-102.

[9] E. Gramage, A. Putelli, M.J. Polanco, C. Gonzalez-Martin, L. Ezquerra, L.F. Alguacil, P. Perez-Pinera, T.F. Deuel, G. Herradon, The neurotrophic factor pleiotrophin modulates amphetamine-seeking behaviour and amphetamineinduced neurotoxic effects: evidence from pleiotrophin knockout mice, Addict Biol 15 (2010) 403-412.

[10] E. Gramage, L. Rossi, N. Granado, R. Moratalla, G. Herradon, Genetic inactivation of pleiotrophin triggers amphetamine-induced cell loss in the substantia nigra and enhances amphetamine neurotoxicity in the striatum, Neuroscience 170 (2010) 308-316.

[11] N. Granado, S. Ares-Santos, R. Moratalla, Methamphetamine and Parkinson's disease, Parkinsons Dis 2013 (2013) 308052.

[12] G. Herradon, C. Perez-Garcia, Targeting midkine and pleiotrophin signalling pathways in addiction and neurodegenerative disorders: recent progress and perspectives, Br J Pharmacol 171 (2014) 837-848.

[13] A. Higuera-Matas, M.L. Soto-Montenegro, G.L. Montoya, V. Garcia-Vazquez, J. Pascau, M. Miguens, N. Del Olmo, J.J. Vaquero, C. Garcia-Lecumberri, M. Desco, E. Ambrosio, Chronic cannabinoid administration to periadolescent rats modulates the metabolic response to acute cocaine in the adult brain, Mol Imaging Biol 13 (2011) 411-415. 
[14] C. Huang, P. Mattis, C. Tang, K. Perrine, M. Carbon, D. Eidelberg, Metabolic brain networks associated with cognitive function in Parkinson's disease, Neuroimage 34 (2007) 714-723.

[15] Y.H. Huang, S.J. Tsai, T.W. Su, C.B. Sim, Effects of repeated high-dose methamphetamine on local cerebral glucose utilization in rats, Neuropsychopharmacology 21 (1999) 427-434.

[16] D.P. Jang, H.K. Min, S.Y. Lee, I.Y. Kim, H.W. Park, Y.H. Im, S. Lee, J. Sim, Y.B. Kim, S.H. Paek, Z.H. Cho, Functional neuroimaging of the 6-OHDA lesion rat model of Parkinson's disease, Neurosci Lett 513 (2012) 187-192.

[17] S.J. Kim, I.K. Lyoo, J. Hwang, Y.H. Sung, H.Y. Lee, D.S. Lee, D.U. Jeong, P.F. Renshaw, Frontal glucose hypometabolism in abstinent methamphetamine users, Neuropsychopharmacology 30 (2005) 1383-1391.

[18] Y.T. Kim, S.W. Lee, D.H. Kwon, J.H. Seo, B.C. Ahn, J. Lee, Dose-dependent frontal hypometabolism on FDG-PET in methamphetamine abusers, J Psychiatr Res 43 (2009) 1166-1170.

[19] I.N. Krasnova, B. Ladenheim, J.L. Cadet, Amphetamine induces apoptosis of medium spiny striatal projection neurons via the mitochondria-dependent pathway, FASEB J 19 (2005) 851-853.

[20] I.N. Krasnova, B. Ladenheim, S. Jayanthi, J. Oyler, T.H. Moran, M.A. Huestis, J.L. Cadet, Amphetamine-induced toxicity in dopamine terminals in CD-1 and C57BL/6J mice: complex roles for oxygen-based species and temperature regulation, Neuroscience 107 (2001) 265-274.

[21] M. Laruelle, Imaging synaptic neurotransmission with in vivo binding competition techniques: a critical review, J Cereb Blood Flow Metab 20 (2000) 423-451.

[22] M.J. LaVoie, J.P. Card, T.G. Hastings, Microglial activation precedes dopamine terminal pathology in methamphetamine-induced neurotoxicity, Exp Neurol 187 (2004) 47-57.

[23] J.F. Marshall, S.J. O'Dell, Methamphetamine influences on brain and behavior: unsafe at any speed?, Trends Neurosci 35 (2012) 536-545.

[24] N.R. McFarland, S.N. Haber, Thalamic relay nuclei of the basal ganglia form both reciprocal and nonreciprocal cortical connections, linking multiple frontal cortical areas, J Neurosci 22 (2002) 8117-8132.

[25] H. Mizuma, M. Shukuri, T. Hayashi, Y. Watanabe, H. Onoe, Establishment of in vivo brain imaging method in conscious mice, J Nucl Med 51 (2010) 10681075.

[26] J. Obrocki, A. Schmoldt, R. Buchert, B. Andresen, K. Petersen, R. Thomasius, Specific neurotoxicity of chronic use of ecstasy, Toxicol Lett 127 (2002) 285297.

[27] M.A. Parvaz, N. Alia-Klein, P.A. Woicik, N.D. Volkow, R.Z. Goldstein, Neuroimaging for drug addiction and related behaviors, Rev Neurosci 22 (2011) 609-624.

[28] J. Pascau, J.D. Gispert, M. Michaelides, P.K. Thanos, N.D. Volkow, J.J. Vaquero, M.L. Soto-Montenegro, M. Desco, Automated method for smallanimal PET image registration with intrinsic validation, Mol Imaging Biol 11 (2009) 107-113.

[29] C.G. Radu, C.J. Shu, S.M. Shelly, M.E. Phelps, O.N. Witte, Positron emission tomography with computed tomography imaging of neuroinflammation in experimental autoimmune encephalomyelitis, Proc Natl Acad Sci U S A 104 (2007) 1937-1942. 
[30] J.K. Roh, H. Nam, M.C. Lee, A case of central pontine and extrapontine myelinolysis with early hypermetabolism on 18FDG-PET scan, J Korean Med Sci 13 (1998) 99-102.

[31] N. Sailasuta, O. Abulseoud, M. Hernandez, P. Haghani, B.D. Ross, Metabolic Abnormalities in Abstinent Methamphetamine Dependent Subjects, Subst Abuse 2010 (2010) 9-20.

[32] K. Shimoji, L. Ravasi, K. Schmidt, M.L. Soto-Montenegro, T. Esaki, J. Seidel, E. Jagoda, L. Sokoloff, M.V. Green, W.C. Eckelman, Measurement of cerebral glucose metabolic rates in the anesthetized rat by dynamic scanning with $18 \mathrm{~F}$ FDG, the ATLAS small animal PET scanner, and arterial blood sampling, J Nucl Med 45 (2004) 665-672.

[33] L. Sokoloff, M. Reivich, C. Kennedy, M.H. Des Rosiers, C.S. Patlak, K.D. Pettigrew, O. Sakurada, M. Shinohara, The [14C]deoxyglucose method for the measurement of local cerebral glucose utilization: theory, procedure, and normal values in the conscious and anesthetized albino rat, J Neurochem 28 (1977) 897916.

[34] M.L. Soto-Montenegro, J. Pascau, M. Desco, Response to Deep Brain Stimulation in the Lateral Hypothalamic Area in a Rat Model of Obesity: In Vivo Assessment of Brain Glucose Metabolism, Mol Imaging Biol (2014).

[35] M.L. Soto-Montenegro, J.J. Vaquero, C. Arango, G. Ricaurte, P. GarcíaBarreno, M. Desco, Effects of MDMA on blood glucose levels and brain glucose metabolism, Eur J Nucl Med Mol I 34 (2007) 916-925.

[36] Y.H. Sung, S.C. Cho, J. Hwang, S.J. Kim, H. Kim, S. Bae, N. Kim, K.H. Chang, M. Daniels, P.F. Renshaw, I.K. Lyoo, Relationship between N-acetyl-aspartate in gray and white matter of abstinent methamphetamine abusers and their history of drug abuse: a proton magnetic resonance spectroscopy study, Drug Alcohol Depend 88 (2007) 28-35.

[37] N.D. Volkow, L. Chang, G.J. Wang, J.S. Fowler, D. Franceschi, M. Sedler, S.J. Gatley, E. Miller, R. Hitzemann, Y.S. Ding, J. Logan, Loss of dopamine transporters in methamphetamine abusers recovers with protracted abstinence, $\mathrm{J}$ Neurosci 21 (2001) 9414-9418.

[38] N.D. Volkow, L. Chang, G.J. Wang, J.S. Fowler, D. Franceschi, M.J. Sedler, S.J. Gatley, R. Hitzemann, Y.S. Ding, C. Wong, J. Logan, Higher cortical and lower subcortical metabolism in detoxified methamphetamine abusers, Am J Psychiatry 158 (2001) 383-389.

[39] B.K. Yamamoto, M.G. Bankson, Amphetamine neurotoxicity: cause and consequence of oxidative stress, Crit Rev Neurobiol 17 (2005) 87-117.

[40] B.K. Yamamoto, A. Moszczynska, G.A. Gudelsky, Amphetamine toxicities: classical and emerging mechanisms, Ann N Y Acad Sci 1187 (2010) 101-121. 


\section{FIGURE LEGENDS}

Figure 1. A) TH-immunostained striatal sections of mice, 4 days after administration of saline (SAL) or amphetamine (AMPH) in PTN-/- and WT mice. The photomicrographs illustrate amphetamine-induced TH loss. The graph shows OD measured in arbitrary units (au) of $\mathrm{TH}$ - immunoreactive fibers in the striatum. Data are shown as mean $\pm S D$ ( $n=5-6$ animals per group). ${ }^{* *} p<0.01$ vs. SAL. ${ }^{* * *} p<0.001$ vs. SAL. ${ }^{\#} p<0.05$ vs. WT. Scale bar $=200 \mu \mathrm{m}$. B) PET study in the striatum of PTN-/- and WT mice after administration of saline or amphetamine. $\left[{ }^{18} \mathrm{~F}\right]-\mathrm{FDG}-\mathrm{PET}$ overlaid on the MRref revealed differences in brain glucose metabolism in the striatum. The graphs show the ratio to WB in the striatum, prefrontal cortex, thalamus and cerebellum. Data are shown as mean $\pm S D$ ( $n=5-6$ animals per group). ${ }^{\#} p<0.05$ vs. WT, ${ }^{\#} p<0.01$ vs. WT. C) $\left[{ }^{18} \mathrm{~F}\right]-\mathrm{FDG}$ uptake: absolute value of SUV, ratio-to-WB and ratio-to-CB for each $R O I$ in the four groups of study. Data are shown as mean $\pm S D$ ( $n=5-6$ animals per group). ${ }^{\#} p<0.05$ vs. WT, ${ }^{\#} p<0.01$ vs. WT. 